\title{
Index
}

Note: In general, spelling and descriptions follow the authors' choices and the respective occurences in the contributions. In some instances, where the English standardised version is preferred or when different versions occur in the contributions, alternative options are stated as well.

\section{Proper Names}

Aaron (OT)/Hārūn (Qur’an) 321, 470-471, 476

'Abbāsid/Abbasids, dynasty 318, 322, 333, 421, 633, 635

'Abd Allāh/Abdullah, emir of Córdoba 331

'Abd Allāh b. 'Amr b. al-'Āṣ 406

'Abd Allāh b. Wahhāb 327

'Abd al-Ghanī Maḥmūd 'Abd al- 'Āṭī 418

‘Abd al-Ḥamīd, Iḅn Ḥabīb’s teacher 327

'Abd al-Malik, Umayyad caliph 320, 410

'Abd al-Malik b. Ḥabīb 13, 319-333, 349

'Abd al-Rahman I, Umayyad emir 331

'Abd al-Raḥman II, Umayyad emir 321, 332

'Abdallāh b. (al-)‘Abbās 637-638

'Abdallāh b. Ḥamza 415, 417-419, 421, 424, 430-434, 438

Abdel Haleem/'Abd al-Ḥalīm 67, 69, 81

Abel (OT) 265, 705, 710, 719-720, 750

Abjesous the Teacher 183

Abraham (OT)/Ibrāhīm (Qur’an) 66, 195, 219, 263, 271, 297-298, 304, 313-314, 324, 403, 469-470, 472-474, 481, 555, 638, 685, 703, 705, 708, 715, 751, 761

Abū 'Abd Allāh Muḥammad b. Muḥammad b. Idrisī al-Qurțubī 398

Abū Bakr 'Abd al-Razzāq b. Hammām al-

Șan'ānī 405-406, 637

Abu Bakr al-Baydak, companion of Iḅn Tūmart 283

Abū Firās b. Di'tham 417-418

Abū Ḥāmid al-Ghazālī 67, 74-77, 79-81, 416

Abū Hurayra 406

Abū l-‘Abbās al-Ṣaffāḥ, first Abbasid caliph 633

Abū l-'Alā' al-Ma'arrī 73

Abū l-Fidā’ b. Kathīr/Ibn Kathīr 72-73, 394

Abū l-Qāsim b. Ḥawqal 397-398

Abū Muḥammad 'Abdallāh al-Bashīr al-

Wansharīsī 283
Abū Ṭālib b. 'Abd al-Muțțalib, Muḥammad's uncle 331

Adah, wife of Lamech (OT) 266

Adam, (OT)/Ādam (Qur'an) 53-54, 144, 199, 212-213, 215, 217, 219, 260, 262, 265-266, $269,271,319,323-324,406,467,469-470$, 473-474, 476, 481, 566, 676, 700, 703, 705, 707, 750

Adomnán, abbot of Iona 652

Adso, monk of Montier-en-Der 28, 30, 199, 231

Aethicus Ister 28, 266

Agamben, Giorgio 16, 733, 736-749, 754-756, 772, 801-802

Agathias 85

Agus, Aharon, rabbi 765, 770

Ahmad, mystic 74

Aḥmad b. Ḥanbal 471

Aḥmad b. Șāliḥ b. Abī l-Rijāl 418

Aḥmed Bīcān 409

Ajāmila, brahmin 620

Ajātaśatru, king of Magadha 592

Akbar, Moghul emperor 624-625

Akșobhya, Buddha 149-151, 378

al-Bīrūnī 184

al-Bukhārī 475

al-Ḍāmī, teacher 428

al-Damīrī 394

al-Hādī ilā al-Ḥaqq Yaḥyā b. al-Ḥusayn, imam 423, 425

al-Ḥākim, Fatimid caliph 284

al-Ḥasan al-Rașșāṣ 429

al-Iskandar, al-Iskander see Alexander the Great 325, 394, 401-402, 407

al-Khūshānī 321

al-Kisā'ī 472

al-Manṣūr 'Abdallāh b. Ḥamza, imam 415, 417419, 422, 424, 430-434, 438

al-Murtaḍā, imam of Yemen 425 
al-Masị̣̄ al-Dajjāl see Dajjāl 5, 402, 405, 474

al-Mu'tamid, caliph 634

al-Mu'tașim, Abbasid caliph 312

al-Nāṣir li-Dīn Allāh, Abbasid caliph 425

al-Qāọī Ja'far b. 'Abd al-Salām 424

al-Qāsim b. Ibrāhīm al-Rassī 425

al-Raḥmān (rahmaṇāvatāra) 144

al-Șa‘b b. dhī Marāthid 638-639

al-Shahrastānī 404

al-Ṭabarī 322-324, 327

al-Tha'labī 371, 472

al-Tirmidhī 302

al-Walīd ị̣n 'Abd al-Malik iḅn Marwān, Umayyad caliph 319, 329, 633

al-Wāqidī, Mohammed ị̣n 'Umar ị̣n Wāqid 327

al-Wātiqq, Abū Isḥāq Muḥammad ị̣n, Abbasid

caliph 16, 634-635

'Alā' iḅn Mughīt 332

Alcuin of York 94, 341-343, 517-518, 679

Aldebrand, Étienne, (arch)bishop 458

Alexander, bishop of Alexandria 52

Alexander the Great/'al-Iskander/Dhū lQarnayn 69, 199-200, 220, 223, 300-301, 325, 390, 392-394, 398, 400-405, 407-408, 411, 631-632, 634-638, 640

Alexander the Rūmì see Alexander the Great 638

Alfons III, king of Asturias 349

Alfred I, Anglo-Saxon king 557

Ali Amir-Moezzi, Mohammad 279, 281

'Alī b. Abī l-Ḥazm Iḅn al-Nafīs 67, 77-81

'Alī iḅn Ḥasan al-Ḥūfĩ al-Qāsimī 398, 422

'Alī Muḥammad Zayd 423-424, 426, 430-431, 433

Almoravids, dynasty 282

Altenstein, Peter Hermann 807

Amalarius of Metz, bishop 721

Amānat Rāy 625

Ambrose, bishop of Milan 342, 684, 700

Amīr Nūr al-Dīn al-Ḥasan b. Yaḥyā b. 'Abdallāh b. al-Hādī ilā al-Haqq 430

Amitābha (Skt)/Amida (Jpn), Buddha 139-140, 149, 151, 286, 378, 382, 536, 541, 580

Amitāyus see Amitābha 536, 543, 580

Anastasios Lolos 199

Anastasios of Sinai, monk 55, 97, 605

Andrew, apostle (NT) 53, 56, 282, 284, 752

Angenendt, Arnold 488-489

Anselm of Laon 33
Anselm, bishop of Havelberg 92

Anshi, empress consort of Japan see below s.v. Fujiwara 530-532, 535-536

Anthony, monk 217

Antichrist 5, 14, 30-31, 36, 69, 186, 199-200, 220-223, 230-231, 278, 281, 284, 286, 337, $339-340,342,345-348,351,402,404-405$, 442, 444-446, 448-449, 460, 467, 474, $482,484-485,487,679,705,709,718-720$, 788,801

Antiochus IV, Seleucid king 278

Apollo, Greek deity 86

Aranemin, king 153

Arendt, Hannah 764

Arfaxat/Arpachshad (OT) 228, 264

Aristoteles/Aristotle 77, 638

Arius, presbyter of Alexandria 342

Arnald/Arnold of Villanova 442, 456

Artaud, Antonin 766

Āryaśūra, Buddhist poet 124, 135

Ashinās, Turkish general 635

Ashkenaz (OT) 390

Assmann, Jan 46

Aśvaghoșa, Buddhist poet 124

Aśvatthāman, mythological character 146

Athanasios I, Byzantine emperor 85

Athanasius, patriarch of Alexandria 45, 48-52, 60

Athenagoras of Athens 495

Atlas, mythological character 187

Audoin, bishop of Rouen 652

Audradus Modicus 29

Augustine, bishop of Hippo 26-29, 49, 92, 184, 206, 212, 217, 266, 287, 337, 342, 350, 480, 492-493, 495, 499, 557, 569, 674, 676-680, 684, 689-690, 700, 702-704, 706-707, 709-711, 716, 769, 773

Augustus, Roman emperor 677-678, 682-687, 689, 691

Avalokiteśvara, Bodhisattva 138, 378, 381, 591

Avicenna/Abū 'Alī al-Husayn b. 'Abd Allāh b. Sīnā 77

'Awf b. Mālik, contemporary of the prophet Muhammad 307, 320

Ayogpa, clan 380

Ayyubids, dynasty 77, 283, 421-422

Azrael/'Izrā'îl (OT) 625-626

Athelbert, king of Kent 549-550, 552-553, 566, 572 
Baeck, Leo, rabbi 765-766

Bahman Isfandiyād/Esfandiyār, king 403

Bahrām II, Sasanian king 186

Bal, Mieke 766

Balaam (OT) 171, 760

Balthasar, Hans Urs von 760, 798

Balthild, Merovingian queen 652

Baraies the Teacher, Mani's disciple 183

Barasch, Moshe 770

Barcelaicus, abbot 649-650

Barnabas 482

Barth, Karl 27, 760

Bartholomew/Bartholomy, apostle (NT) 53-54, 56

Bartholomew, Peter 283

Bashear, Suliman 304, 306-307, 309

Basil the Younger 611, 613

Bataille, Georges 766

Baudrillard, Jean 271

Beatus of Liébana, monk 29, 342, 647, 651

Beaufret, Jean 783

Bede the Venerable/Beda Venerabilis, monk 16, 551, 554, 557-558, 560, 563, 642, 645, 655, $658,682,698-702,704-707,711,713-719$, 722

Bengel, Albrecht Johan 790

Benjamin, Walter 736, 759, 761, 777, 801-802

Benz, Maximilian 256

Beowulf, legendary hero 556

Berkouwer, Gerrit Cornelis 188

Bernard, abbot of Clairvaux 36-37, 39

Bernhard, duke of Septimania 511

Bhāviveka/Bhavya, Buddhist philosopher 154, 157

Bimbisāra, king of Magadha 150, 592-593, 598-599

Bischoff, Bernhard 203-204

Bishtāsaf/Bishtasb 403-404

Bloch, Ernst 769

Blumenberg, Hans 272, 786, 794

Bodhisattva, Indian Buddhist author 135, 138, $145,150,152,363,368,377,536,538,540$, 590-591

Bodo-Eleazar, deacon 345, 347

Bogyay, Thomas von 88, 93, 99

Böhme, Hartmut 787

Bollack, Jean 786, 807-808

Boniface, Anglo-Saxon missionary 563

Boniface VIII, pope 445, 449, 452
Botschko, Moshe, rabbi 760

Botschko, Yerahmiel Eliyahou, rabbi 760

Bourdieu, Pierre 786

Bowersock, Glen 281

Boyarin, Daniel 771

Brahma/Brahmā, Hindu deity 539-540, 620

Brentano, Margherita von 766

Bstan ma bcu gnyis, the twelve goddesses of

Tibet 374

Bu ston Rin chen grub, abbot 364

Buddhaghosa, Buddhist exegete 589

Bukht Naṣr see Nebuchadnezzar 403, 405, 631

Bultmann, Rudolf 27, 182-183

Busche, Jürgen 778

Bush, George W. 289

Bya bral Rin po che, author (Sangs rgyas rdo

rje) 366,382

Byang chub seng ge 143

Byzantia, daughter of Byzas and Chouseth 200

Byzas, Byzantine king 200

Caesar, Gaius Iulius 685-687, 689, 717, 778, 799

Caesarius, bishop of Arles 209-213, 499, 715

Cain (OT) 199, 265-269, 566, 750-751

Cainan, descendant of Seth (OT) 265

Cainites, dynasty (OT) 266-269

Cakravartin, the wheel-turning monarch in Buddhism 134, 153, 377

Caṇdapradyota, king 134

Candragarbha, Bodhisattva 363

Cathwulf, member of the House of Wessex 522

Celestine V, pope 445, 447

Chado, bishop 649-650

Charlemagne, Carolingian king and emperor 30 , 58, 205, 341, 510, 676, 679, 698, 712, 723

Charles I/Charles of Anjou 452

Charles the Bald, Carolingian king and emperor 344, 511

Childebert, Merovingian king 651

Childeric II, Merovingian king 651-653

Chilperic, Merovingian king 484-485

Chlothar III, Merovingian king 647, 650-652

Chouseth, wife of Byzas 200, 220

Christian of Stavelot, monk 674-677, 680-682, 684, 686-687, 690-691

Cioran, Emil/Émile 770, 786

Citragupta, warden of hell 618

Claudius, bishop of Turin 681

Clemens, Irish missionary 563 
Clement VI, pope 443, 445

Clement of Alexandria 499, 562, 607

Clothar III, Merovingian king 647, 649-653

Clovis/Chlodovech, Merovingian king 651

Colli, Giorgio 783

Columbanus, Irish missionary 642, 655

Constantine, consul 650

Constantine I, Roman emperor 57, 764

Constantine III, Byzantine emperor 611

Constantius II, Roman emperor 94

Corbin, Henry 786

Cromwell, Oliver 769

Crowley, Aleister 271

Cyril, patriarch of Jerusalem 94, 443, 447

Cyrinus, governor of Syria 683

Cyrus the Great, Persian emperor 716-717

Dajjāl/Djaddjāl, Antichrist in Islam 281, 474475

Dākhil see Dajjāl 69, 281, 284, 312, 331, 404

Damian, saint 87, 91, 108, 569

Dan, tribe 221

Daniel (OT) 2, 28, 30, 97, 165, 167-168, 175, 347, 442, 444, 553, 680, 768

Dārā Shukoh, Moghul prince 624

David (OT) 99, 208, 326, 329, 469, 471-473, 685, 703, 705-706, 708, 715, 762

Decius/Daqiyānūs, Roman emperor 169, 410

Dee, John 270-271

Deleuze, Gilles 783

Dempf, Alois 765

Derrida, Jacques 770, 786-787

dGe bsnyen gLe ru, Tibetan mountain-god 374, 378

Dharmakāya, one of the three buddhabodies 381-382

Dhū 'l-Qarnayn, Qur'anic hero see Alexander the Great 393-394, 401-403, 408, 411, 634, 638-640

Dhuoda of Septimania 15, 509, 511-523

Diadochi 755

Dionysius, bishop of Alexandria 49, 56

Dionysius Exiguus, monk 567, 660, 666

Dodanim (OT) 390

Dōkyō, Japanese Buddhist monk 535-536

Domitian, Roman emperor 169

Domnall mac Áedo of Cenél Conaill, king of northern Ireland and western Scotland 646

Dōmyō, Japanese Buddhist monk 539-540

Dryhthelm 557-558
Dulkarnèn, angel see arab. Dhū l-Qarnayn 393394

Eadwine, king of Northumbria 551

Eddius Stephanus 551

Edelsteins, family 760

Eichhorn, Gottfried Johann 790

Ekkehard of Aura, monk 287

Elam, grandmother of Rasu'eja 264

Elchasai/Elchasaios, prophet 181-184

Eleazar (OT) 278

Eleazar, rabbi 774

Elijah, prophet (OT) 200, 221, 760

Elipandus, bishop of Toledo 342-343

Elisabeth of Schönau 37

Elisha(h) 494, 502

Elishah (OT), son of Javan 390

Engels, Friedrich 790

Enkelados, giant 94

Enoch (OT) 12, 45, 50-51, 186, 200, 221, 255267, 270-272

Enoch-Metatron 260

Enos, descendant of Seth (OT) 265

En'yū Tennō, Japanese emperor 532

Erkenwald, saint 564

Eshin Sozu see Genshin 539

Eucherius, bishop of Lyon 214-215

Eulogius, bishop of Córdoba 345-346, 348349

Eusebius of Alexandria 58

Eusebius, bishop of Caesarea 49, 56, 480-482, 492, 645, 684, 702

Eustratius of Constantinople 556

Eve (OT) 219, 260, 262, 265, 313, 750

Ezekiel (OT) 2, 86, 91, 165, 167-168, 172, 175, 220, 337, 350, 410, 493, 502

Farinie de Gourdon, Guillaume, inquisitor 442

Fāțima 331

Fatimids, dynasty 421

Felix, bishop of Urgell 343

Fenrir, the wolf in Scandinavian mythology 568-569

Ferreolus, martyr 496

Feuerbach, Ludwig 578, 597

Finkelstein, Louis 765-766

Flavius Josephus 265-267, 269, 271, 400, 632, 700,717

Fortunatianus, bishop of Aquileia 684

Foucault, Michel 16-17, 733, 736, 765, 783788, 792-796, 800-810 
Frechulf, bishop of Lisieux 682

Fredegar 16, 646-647, 699

Frederick II, Holy Roman emperor 444

Frederick III, Holy Roman emperor 444

Fredurichus, bishop of Utrecht 681

Freud, Sigmund 784, 795

Fujiwara, Japanese aristocratic family 286, 530 Fujiwara no Anshi (also called Yasuko), imperial consort 530

Fujiwara no Koremasa, house elder 538

Fujiwara no Michinaga, house elder 530

Fujiwara no Sanesuke, house elder 533

Fulgentius, bishop of Ruspe 684

Fursey, Irish monk 557

Gabriel, archangel (OT) 54-55

Galenos/Galen 638

Gaṇeśa, Hindu deity 145

Gao Qiao, Buddhist disciple 590-591

Gasché, Rodolphe 770

Gautama Siddhārtha, the historical

Buddha 154, 369

Gennadius of Marseille, presbyter 215

Genshin or Eshin Sozu, Tendai cleric 539

Gente, Peter 808

Georgios Monachos 611

Gerberga, Frankish queen 30

Germanus, bishop of Auxerre 490

Gibbon, Edward 281

Gideon (OT) 199

Gildas 211, 642-644, 667

Godfrey of Bouillon, duke of Lorraine 33

Gog 444

Gog and Magog/Ya'jūj wa-Ma'jūj 5, 14-16, 69, $198,200,220,227,300,325,337,350,390-$ 394, 396-403, 405, 407-411, 631-640, 803 Gomer (OT) 390-392

Gongsun Qiang, official in the underworld 581, 591

Gotfrid, ruler of the Alemans 206

Gregory, bishop of Langres 488

Gregory, bishop of Nyssa 93, 604-605

Gregory, bishop of Tours 14, 340, 479-495, 497-503, 663-666

Gregory, disciple of Basil the Younger 611

Gregory of Nazianzus, archbishop of Constantinople 684,796

Gregory the Great, pope 208, 210, 212, 343, $347,499,549-550,552,555-557,559-560$, $563,566,572,684,707$
Gregory VII, pope 289

gTer dbon Nyi ma seng ge, Tibetan lama 359, 379, 388

gTer ston bZang po grags pa see Ri khrod pa bZang po 367

gTer ston 0 rgyan mchog gyur gling pa 380

Gundovald, ruler of Aquitaine 489-490

Guntlandus, monk 681

Gupta, Indian dynasty 1, 137, 151

Gushtāsp 404

Habermas, Jürgen 479, 809

Hadrian I, pope 205

Hagar (OT) 195, 219, 350

Haggai Ben-Shammai 300

Haimo of Auxerre, monk 228, 680

Haistulf, archbishop of Mainz 681, 684

Ham, Noah's son (OT) 470

Hamīd al-Dīn, dynasty 422

Han, dynasty 581, 584-586, 590, 596

Hārūn, Iḅn Ḥabīb's brother 321

Ḥasān Iḅn Abī l-Ḥasan al-Daylamī 410

Ḥassān b. Thābit 639

Ḥătimids, dynasty 421

Hegel, Georg Wilhelm Friedrich 736, 745-746, 749, 769, 785, 798-799, 802, 804

Heidegger, Martin 735, 768, 798, 804

Hemacandra, Śvetāmbara Jaina Polymath 126

Henry VI, king of England 454

Heraclius, Byzantine emperor 296, 330, 400

Herod I, king of Judea 485, 566, 709

Hesiod 123, 258

Hilarius, pope 654

Hildegard von Bingen, abbess 37, 270

Hippocrates 77

Hippolytus of Rome 189, 231, 482, 645

Hohenstaufen, dynasty 444

Hölderlin, Friedrich 749, 791

Hōnen, Japanese Buddhist theologian 150, 286, 536

Hormizd II, Sasanian king 186

Hrabanus Maurus, abbot of Fulda 30, 647, 677, 681-682, 684-687, 689, 691

Hübener, Wolfgang 807-808

Hugh of Fleury, monk 691

Ḥumayd b. Ạ̣mad al-Muhallī 432

Ḥunayn b. Isḥāq 636

labal, son of Lamech (OT) 266

Iḅn Abbās 327, 331

Iḅn al-Athir 283, 631 
Iḅn Ḥabīb see ‘Abd al-Malik b. Ḥabīb 13, 319333, 349

Iḅn Hishām 401-402, 637-639

Iḅn Ḥumaydī 330

Iḅn Isḥāq 322, 401-402

Iḅn Kathīr see Abū l-Fidā' b. Kathīr

Iḅn Khurdādhbih 634

Iḅn Ṭāwūs 405

Iḅn Tūmart 282-284

Iḅn Tyfayl/Tufayl 78

Ibrāhīm al-Khalīl, the "friend of God" see

Abraham 144, 403

Idrīs, Islamic prophet 470

Ignatius, bishop of Antioch 176, 492, 771

Imru' al-Qays 638

Indra, Hindu deity 539, 588-590, 597, 618

Innaios, brother of Zabed 183

Innocent VI, pope 447

Irad, descendant of Cain (OT) 266

Irenaeus, bishop of Lyon 492

'Īṣ b. Isḥāq b. Ibrāhīm 638

'Isā b. Miryam/Jesus (Qur'an) 324, 405, 409410, 638

Isaac (OT), Isḥāq (Qur’an) 263, 638

Isaac, rabbi 774

Isaiah (OT) 28, 45, 50-51, 69, 91, 165, 175, 279, 406, 456

Isebel (OT) 171

Ishmael (OT)/Ismā‘īl (Qur’an) 199-200, 219,

221, 350, 403, 405

Ishmaelites (OT) 195, 197, 200, 219-223, 338,

347-348

Isidore, bishop of Seville 219, 226, 228, 342,

350, 396, 522, 646-647, 649, 653, 700, 719

Iskandar, sultan 394

Islamic State of Iraq and the Levant (ISIL) 284,

305, 309, 312, 330

Ismā‘īl al-Muzayyin 428

lubal, Lamech's son (OT) 266-267

Izumi Shikibu 539-540

Jacob (OT) 469-470, 686, 708, 760-761

Jacob, Jewish merchant 297

Ja'far b. al-Imām al-Qāsim b. 'Alī al-‘lyānī 436

Jagajjaya Malla, ruler of Kathmandu 380

James, apostle (NT) 53

Japheth, Noah's son (OT) 219, 390, 392

Jared, descendant of Seth (OT) 265

Javan (OT) 390
Jean de Roquetaillade see John of Rupes-

cissa 39

Jeremiah/Jeremy (OT) 28, 30, 69, 175, 337, 345, 408,716

Jeroboam (OT) 445

Jerome, church father $29,50,203,212,217$, $231,337,342,347,480,482,492,495,682$, 684,700

Jesus Christ 49, 51-53, 69, 90, 165, 181, $183-$ 184, 186-187, 189-190, 215, 266, 281, 299300,312 , 322, 324, 342, 402, 405-406, 409411, 449, 451, 458, 467-468, 470, 473-476, $515,519,638,649-651,675,682,687-688$, $703,711,751-754,756,762,767-769,771-$ 772, 775-776, 788 see 'Isā b. Miryam

Jetsun Dampa 125

Jien, Tendai abbot 286-288

'Jigs med gling pa, yogi 368

Jina, founder of Jainism 125

Joachim of Fiore, monk 5, 11, 29, 37-39, 92, $446,450,769,799$

Job (OT) 347

Johannes, abbot of St. Gall 213

John, apostle (NT) 37, 53-54, 278, 655, 752-753

John, author of the Apocalypse 52, 91, 165-176, $278,559,711,733,752,755,767$

John II, king of France 456, 460

John Chrysostom, bishop of Constantinople 217, 684

John of Rupescissa/Jean de Roquetaillade, monk 14, 441-442, 451, 458

John of Salisbury 38

John of Seville 346

John Rufus, priest 97, 649-650

John Scotus Eriugena 681

John the Almgiver, patriarch of Alexandria 611

John the Baptist 96, 98, 100, 490, 561, 752

Jonas of Orléans 522

Jonas, Hans 764, 766, 798, 800

Jonitus, Noah's son (OT) 199, 219, 223

Joseph (OT) 471-472, 708, 715

Joshua (OT) 471

Joshua ben Levi, rabbi 775

Judah, kingdom 762

Judas (OT) 278, 566

Julian of Brioude 501

Julianus Africanus 645

Julius Africanus 189, 482

Jupiter, Roman deity (Greek Zeus) 144, 211 
Justin Martyr 57

Justinian I, Byzantine emperor 29, 59

Justus, archbishop of Canterbury 297

Ka'b al-Aḥbār 638

Kabīr, Hindu saint and poet 621-622

Kafka, Franz 765-766, 770, 775

Kainam, son of Rasu'eja (OT) 264-265

Kalki(n), Hindu messianic figure 1, 5, 143-146, 154

Kalkin Cakrin Buddhist messianic figure: 2, 145-146

Kāma, the Indian Eros 369

Kaṇāda, founder of the Vaiśeșika system of philosophy 137

Kant, Immanuel 748, 769, 809

Kantorowicz, Ernst 741

Kapila, legendary founder of the Sānkhya system of philosophy 137

Kaurava(s), legendary Indian family 137

Kauțilya, Sanskrit author 133

Kerényi, Karl 786

Khosroes II, Persian king 400

Khri srong Ide btsan, Dharma king 367-368, 371, 375, 377

Khwarizm Shah, dynasty 631

Kierkegaard, Søren 769

Kimura Taiken 363

Kittim (OT) 390

Klopstock, Robert 775

Klossowski, Pierre 783

Ko Sōnji, Chinese general 633

König, René 759

Koselleck, Reinhart 787-790

Kṛkin, king 147

Ḳ̣nmati Mahdī, Muslim heresiarch in Tantric literature 145-146

Kṛ̣ṇa, Hindu deity 137, 154, 620

Kṣatriya, caste (military aristocracy) 579, 589, 597-598

Kṣemendra, Sanskrit author 154

Kublai Khan, Mongol khagan 370

Kumārila, Hindu philosopher 139-140

Kuroda Toshio 529

Kustaios, Mani's disciple 183, 185-188, 190

Laden, Osama bin 289

Lambert, abbot de Saint-Omer 33

Lamech, descendant of Seth (OT) 265-266

Later Han dynasty (Hou Han shu) 584-586, 597

Lazarus (NT) 753-754, 769
Leites, Edmund 770

Leo, monk 656

Leo I, Byzantine emperor 57

Leo I, pope 654, 659

Lepidus, Marcus Aemilius 686

Lessing, Ephraim Gotthold 769

Leudegard, bishop of Autun 652

Lévi-Strauss, Claude 746

Levinas, Emmanuel 747

Liang, emperor 589, 593

Licinianus of Cartagena 58

Lionman see Narasiṃha 620

Liu Ban 584

Liu Cunren 585

Liu Qiu, lay Chinese Buddhist 586

Louis, king of Sicily 444-446

Louis IV the Bavarian, Holy Roman

Emperor 444, 460

Louis IX, king of France 452, 454

Louis the German, king of East Francia 30

Louis the Pious, emperor 30, 204-206, 345,

511, 680-681, 698, 722

Löwith, Karl 764, 783, 798, 800

Lucifer 446

Lücke, Friedrich 791

Luhrāsaf/Lohrasb, king 403

Luke, apostle (NT) 53, 683-684, 687

Luria, Isaac, rabbi and kabbalist 770

Madai, son of Jafet (OT) 390

Madhumati (/-pati), Sanskrit name of

Muḥammad 144

Magog see Gog and Magog

Mahalaleel, descendant of Seth (OT) 265

Mahmud of Ghazni, ruler 621

Maitreya, the future Buddha 2, 6, 143, 362

Makarios of Egypt 609-610

Ma'mar iḅn Rāshid, teacher of 'Abd al-

Razzāq 405

Mamluks, dynasty 77

Mandodarī, Rāvaṇa's wife 623

Manes, chthonic deities 624

Manfred, king of Sicily 445

Mani, prophet 12, 181-184, 186, 188

Mār Yaqūb of Serūgh 399

Māra, personification of death in

Buddhism 126, 362, 369-370, 372-373, 383

Marcellinus 700

Marcion 799

Marco Polo 397 
Marcuse, Herbert 786

Margalit, Avishai 770

Marius, bishop of Avenches 482

Mark, apostle (NT) 53

Mārkạ̣ḍeya, mythical Hindu sage 620-621

Marpa, Tibetan Buddhist teacher 143

Marthad the Younger 638

Martin, bishop of Tours 481, 500

Marx, Karl 769, 784, 798, 804

Mary (NT) 91, 96, 98, 100, 312, 342, 402, 405, 472, 475, 559, 605, 638

Marzūq b. Yaḥyā al-Jawrī/al-Ḥawrī 433

Maslama, original name of Musaylima 417, 432

Mātrceța, Indian Buddhist poet 126, 140

Mattathias (OT) 278

Matthes, Axel 766

Matthew, apostle (NT) 53, 97, 100, 185, 343, 520, 674, 677, 680-685, 691

Maurya, Indian dynasty 137

mChog sgrub lde, king of Gung thang 368

Mehujael, descendant of Cain (OT) 266

Mendelssohn, Moses 809

Mercator, Gerard 397

Meshech (OT) 390-391

Messiah ben David 762

Messiah ben Joseph 762

Metatron, archangel (OT) 260

Methodios/Methodius, bishop of Patara 194, 196, 206, 217-218

Methuselah, descendant of Seth (OT) 265

Metteyya, Pali form of Maitreya, the next

Buddha 362

Metusael, descendant of Cain (OT) 266

Michael, archangel (OT) 33, 36, 47, 54-55, 445 , 568

Migetius, priest 342

Miller, Konrad 398

Miller, William 789

Minucius Felix 492, 495

Miryam (OT)/Maryam (Qur'an) 402

mKhan po Nyi ma don grub, Yolmo lama 366 , 370

mKhas grub rje, Tibetan Buddhist philosopher 126

Montinari, Mazzino 783

Moses (OT)/Mūsā (Qur'an) 45, 48, 50-51, $93-$ 94, 255, 264, 313, 324, 470-471, 473-476, 708, 751, 768, 770-775

Mu‘āwiya, caliph 307, 632-633
Mu dur nag po, Mongolian general 370

Mu khri bTsan po, Tibetan king 367

Muhammad/Mohammed, prophet 2, 4, 13, 67$69,78,80-81,144,281,289,294-304,306-$ 308,312 , 315, 319-324, 345-346, 350, 405, 417, 454, 467-469, 473-477, 634, 639, 773

Muḥammad, amir and son of Ja'far b. al-Imām al-Qāsim b. 'Alī al-'Iyānī 436

Muḥammad I, emir of Córdoba 349

Muḥammad Fațị̣/Futaỵ̣ 433

Muhammad of Ghor, sultan of the Ghurid Empire 621

Mummolus, patricius 489-491

Müntzer, Thomas 769

Murakami Tennō, Japanese emperor 530

Mūsā see Moses

Mūsā iḅn Nuṣayr, governor of North Africa 319 , 327-333, 349

Musallam al-Laḥjī 428

Musaylima al-kadhdhāb/Maslama, contemporary of Muḥammad 417, 432

Musil, Robert 749

Naamah, daughter of Lamech (OT) 266

Nabi Ngisa, prophet 411

Naciketas, brahmin 617-618

Nancy, Jean-Luc 770

Nanda, Indian dynasty 137

Narasiṃha see Nrsiṃha

Nārāyaṇa, Ajāmila’s son, alias of Kṛ̣na 620

Nāropa, Tantric teacher 143

Nashwān b. Sa‘īd al-Ḥimyarī 432, 434, 638-639

Nebuchadnezzar I/Bukht Nașr, king of

Babylon 631

Nephilim, giants (OT) 255-256, 268

Nero, Roman emperor 447, 484-485

Nezamī Ganjāvī 410

Nicetas, patrician 611

Nichiren, Japanese Buddhist theologian 148, 150, 277, 285-286, 288, 538

Nietzsche, Friedrich 769, 776, 783-784, 792, 804,808

Ninus, ruler of Assyria 685

Nirmāṇakāya, one of the three buddhabodies 381

Noah (OT)/Nūh (Qur’an) 199, 219, 223, 254, 256, 262, 264-266, 324-325, 401, 470, 472473, 481, 703, 708, 715

Nṛsiṃha/Narasiṃha incarnation (avatāra) of Viṣnu see Lionman 620 
Nūḥ see Noah

Nu'aym b. Ḥammād, author 304, 332

Nyi ma bzang po, disciple and biographer of Rig 'dzin rGod Idem 367

Nyi ma seng ge 359, 379-382, 388

0 rgyan gling pa, "treasure-finder" in the bKa' thang literature 364-365

'Od dpag med, Tibetan name of the Buddha Amitābha/Amida 382

Okkāka/Skt. Ikṣvāku, king 130

Origen 49, 497, 562

Orosius, Paulus 16, 26, 28, 480, 674-691, 699-700, 717-718

Orsini, family 446

Oswald, king of Northumbria 566

Oterius, monk 342

Otfrid of Weissenburg, monk 681, 687

Otto III, Holy Roman emperor 31, 33

Otto, bishop of Freising 38

Padma gling pa, “treasure-finder" in the bKa' thang literature 364

Padmasambhava, semi-legendary Tantric master 359, 366-368, 371, 376-378, 380, 382-383

Pahlava, Sanskrit name of the Parthians and an invador king 362

Palladius, bishop of Helenopolis 700

Pāṇdava(s), sons of Pandu, legendary Indian family 137

Paschasius Radbertus, abbot of Corbie 674, 677, 681-682, 687-691

Patañjali, Sanskrit grammarian 131

Paul, apostle (NT) 16, 28, 48, 50-51, 53, 85, 95, 171, 173, 184-185, 341, 345, 494, 514, 607, $609,716,733,749,759,764,767-778,799$, 801,805

Paul Albar 345-349

Paul the Deacon, monk 349, 682

Paulinus, bishop of Northumbria 559

Pelagius, pope 486

Penemue (Book of Enoch) 260

Peter, apostle (NT) 53, 56, 95, 458, 559

Peter, interlocutor of Gregory I 556

Peter, translator of Ps.-Methodius 197

Peter of John Olivi, monk 442, 446, 460

Petras, Otto 798

Petrus Comestor 267-268

Petrus Perrier, monk 448

Petrus Riga 266-267, 269
‘Phags pa, Kublai Khan's teacher 370

Philipp, apostle (NT) 53

Philippos, monk 605

Philo of Alexandria, Jewish philosopher 716, 805

Philostorgios, author 94

Pietro Orseolo, doge of Venice 31

Pilate, Pontius 753

Pippin II of Aquitaine 511

Pippin III, the Younger, king of the Franks 667

Pliny the Elder 57, 174

Polycarp, bishop of Smyrna 176

Prajñāvarman, Indian Buddhist author 154-155

Probianus, physician 94

Prometheus, titan 258, 262, 272

Prudentius, bishop of Troyes 345

Ps.-Așma‘ī 402-403

Ps.-Athanasius 55

Ps.-Bede 211

Ps.-Callisthenes 393, 403

Ps.-Ephrem 93, 97, 99-100

Ps.-Hippolytus 231

Ps.-Isidore 210

Ps.-Makarios 609

Ps.-Methodius 85

Ptolemy 397

Qāsim, son of Muḥammad Fațịh 433

Qāsimī, dynasty 422

Qin, dynasty 594, 596

Quinet, Edgar 288

Qutayba, governor of the caliph al-Walĩd 633

Radbod, Frisian ruler 562-563

Radegund, Merovingian queen 480, 483

Ragau, descendant of Shem (OT) 219

Ral pa can, king of Tibet 371-372

Rāma, incarnation (avatāra) of Viṣṇu 137, 154155, 621, 623-625

Rashi, Torah and Talmud commentator 765, 770, 774

Rāshid b. Muḥammad al-Șaqrī al-Janbī, a leading Muțarrifī 417, 433

Rasu'eja, wife of Arpachshad (OT) 264

Ratchis, king of the Lombards 647

Ratnagarbha, a former/previous Buddha 152

Ratnākaraśānti, Indian Buddhist teacher 126

Rāvaṇa, demon king in the Hindu epic

Rāmāyaṇa 623-625

Raymond IV, count of Toulouse 33

Raymond of Aguilers 33, 283-284 
Rædwald, king of East Anglia 553

rDo rta nag po/Go rta nag po, Mongolian general 370

Reccafredus, bishop of Seville 344

Reizei Tennō, Japanese emperor 532

Remigius, bishop of Rheims 681

Remigius of Auxerre, monk 721

Rhodanim (OT) 390

Ri khrod pa bZang po, hermit of Mt. Bkra bzang 367

Ricœur, Paul 27, 786

Rig 'dzin Padma 'Phrin las, abbot 379

Rig 'dzin rGod Idem, founder of the Northern

Treasure tradition 359, 365-368, 370-371,

377, 379-380

Riphath (OT) 390

Roboam (OT) 445

Roger II, king of Sicily 398

Romulus/Romillus 220

Romulus Armelaus 200

Rosenstock-Huessey, Eugen 768

Rosenzweig, Franz 768, 774

R̦̣abha, legendary Indian teacher 137

Rudolf von Ems 267-270

Rudra, Hindu deity 146, 590, 619-620

Rufinus of Aquileia 700

Rufus, Roman consul 649-650

Rūmī 625, 638

Ruppert von Deutz 92

Ryōgen, Japanese Buddhist abbot 537-539

Saichō, Japanese Buddhist theologian 537

Sakhr, jinn 326-327

Saladin arab. Șalāḥ al-Dīn, Ayyubid ruler 631

Sallām, translator 635-637, 640

Salmaios the Ascetic, Mani's disciple 183

Salomon (OT) 708, 711, 715

Samael, archangel (OT) 54-55

Sambhogakāya, one of the three buddhabodies 381

Samudrareṇu, brahmin 152-153

Samuel, archangel (OT) 55

Sangs rgyas bla ma, "treasure-revealer" 365

Sangs rgyas gling pa, "treasure-finder" 364

Sannō Gongen, protector deity of Tendai

Buddhism 537-538

Sarah/Sarai (OT) 263, 350, 469

Sāsān, forefather of the Sasanids 403

Satan 172-173, 271, 284, 324-325, 345, 392, 642,767
Satanael (OT) 55

Saturn, Roman deity 86

Satyaka, an interlocutor of the Buddha 134

Saul (OT) 705, 708, 715

Savonarola, Girolamo, Dominican friar 92

Sayf b. 'Umar 330

Sæberht, king of Essex 553

Schleiermacher, Friedrich 791

Schmitt, Carl 738, 761, 779, 800-801, 804

Scholem, Gershom 761-764, 779, 798

Sebuktigin, founder of the Ghaznavid dynasty 621

Sedulius Scottus 681

Sem (OT) 264

Seneca, Lucius Annaeus 215

Seraphim (OT) 91

Serenus, bishop of Marseilles 560

Sergius, monk 450

Sergius, saint 489, 491

Seth (OT) 265-270

Shakyamuni/Śākyamuni, the historical Buddha 285, 288

Shansud-Din Iliyas/Shamsuddin Ilyas Shah/ arab. Shams al-Dīn, military leader 370

Shinran, Japanese Buddhist monk 150

Shōtoku/Kōken Tennō, Japanese female emperor 535

Shun, Chinese emperor 584

Si tu pan chen, Tibetan scholar 380

Sigebert III, Merovingian king 646

Sigibert I, Merovingian king 481

Sigismund, Burgundian king 501-502

Sisenand, king of the Goths 350

Skanda, Śiva's son 145, 619

Smaragdus, abbot of St. Mihiel 216

sNgags 'chang Śākya bZangpo, "treasurerevealer" 359, 374

Solomon (OT)/Sulaymān (Qur'an), king 319, 321, 326-329, 333, 471, 475, 705

Somadeva, Hindu author 620

Sozomen, Salmasios Hermeias 94

Spengler, Oswald 765, 778

Spes, abbot of Campli 559

Spinoza, Baruch 776

sPyan ras gzigs, Tibetan name of Avalokiteśvara, Bodhisattva 381

Srong btsan sgam po, Tibetan king 371

Stephen, saint 490

Stephen II, pope 667 
Stephen Harding, abbot of Citeaux 33, 36

Sucandra, Bodhisattva 145

Suibne mac Commáin of the Déisi, king of mideastern Ireland 647

Șulayḥids, dynasty 436

Sulaymān b. Muḥammad b. Aḥmad al-Muḥallī, Muțarrifĩ theologian 424-427

Sulaymān iḅn 'Abd al-Malik, Umayyad caliph 329-330

Sulpicius, saint 650

Sulpicius Severus 28, 482, 484

Sūradāsa (Surdas), Hindu author 622

Susan, mother of Rasu'eja 264

Symeon Stylites the Younger 608-609, 612

Szondi, Peter 786-787

Śaka, Central Asian dynasty 362

Śākya, the clan of the historical Buddha 154, 363,369

Śākyamuni, historical Buddha 147-154, 360, 368, 374, 580, 590

Śan̉karasvāmin, Indian Buddhist author 153154

Śāntamati, Bodhisattva 152

Śāntideva, Indian Buddhist author 139

Śăriputra, one of the chief disciples of the Buddha 148

Śiva/Maheśvara, Hindu deity 138, 145-146, $616,618-620,625-626$

Śuddhodana, king of Kapilavastu and father of the historical Buddha 154

Śveta, legendary king (Skandapurāna) 619-620

Šāhbur I, šāh-in-šāh, Sasanian ruler 182

Taiwu, Chinese emperor 586

Tārā, Vālin's wife, queen of Kiskindhya 623

Ṭāriq b. Ziyād, client of Mūsā ị̣n Nuṣayr 319, 327, 329

Tarquinius, Roman king 717

Tarshish (OT) 390

Taubes, Jacob 16-17, 759-761, 763-779, 783788, 797-809

Taubeses, family 760

Tenmu Tennō, Japanese emperor 533

Tertullian 492, 495

Thaddaeus, apostle (NT) 53

Theobald of Étampes 27

Theodora, Byzantine empress 610-613

Theodore, archbishop of Canterbury 211, 554

Theodosius II, Byzantine emperor 29, 57, 498

Theodulf, bishop of Orléans 721
Theophylact of Simocatta 303

Theuderic III, Merovingian king 647, 651-653

Thiébaux, Marcelle 511

Thomas, apostle (NT) 52-53

Thubten Gyatso, Dalai Lama 125

Tiberius, Roman emperor 313

Timotheos, Mani's disciple 183-184

Tiras (OT) 390

Tișya, celestial body 144

Titans, figures in ancient Greek religion 272

Togarmah (OT) 390-391

Tokugawa, Japanese dynasty 541

Tongso, clan 380

Trajan, Roman emperor 57, 563

Trier, Lars von 734

Tubarlak, Persian emperor 301

Tublacain, son of Lamech (OT) 266

Tulasīdāsa/Tulsīdās, Hindu author 623

Tyconius, bishop 92

'Ubayd Allāh b. Ziyād, governor 633

Ubayy b. Ka‘b 299-300

Uda Tennō, Japanese emperor 530

'Umar b. al-Khațțāb, caliph 394

'Umāra iḅn Zayd 407, 410

Umayyads, dynasty 13, 298, 319, 322, 331

Usuard of Saint-Germain-des-Prés, monk 344

'Uthmān, caliph 320, 330

Vaidehī, wife of king Bimbisāra 150

Vaiśravaṇa, Northern Celestial King in

Buddhism 592

Vālin, a monkey king in Hindu mythology 623

Vallabha, founder of the Path of Grace 622

Vālmīki, (semi-)legendary author of the

Rāmāyaṇa 623

Vāsava, a name of the Hindu god Indra 141

Vasubandhu, Indian Buddhist teacher 141, 146, 150-151, 364

Vattimo, Gianni 783

Vergil, Publius Maro 215

Vessavaṇa, Pali name of Vaiśravaṇa/Pali

Vessavana 598

Veyne, Paul 807-808

Victor, bishop of Capua 655

Victorinus 92, 684

Victorius of Aquitaine 480, 646, 649-651, 653655, 657-662, 664-666

Victricius, bishop of Rouen 496-497

Vigilantius of Calagurris, presbyter 497

Vimalakīrti, Buddhist/Bodhisattva layman 591 
Vincent Ferrier, Dominican monk 39

Vincentius, bishop of Ibiza 58

Viṣnu, Hindu deity 145-146, 153-155, 616-623, 625-626

Vișṇuyaśas, name of Kalki(n), Viṣṇu's tenth and last incarnation (avatāra) 144

Vyāsa, (semi-)legendary author of the Mahābhārata 137

Wahb b. Munabbih 637-638

Wahl, Jean 783

Wamba, Visigothic king 647, 649, 653, 666

Wang Jiangfei 589-591

Wei, Chinese state 581

Weininger, Otto 798

Weituoshi, king 592

Wen, Chinese emperor 582, 592

Western Jin dynasty 594

Wilfrid, bishop of Northumbria 551

William of Septimania, count 511-512, 515-516, 518-523

William IV, duke of Aquitaine 31

Willibrord, missionary 563

Winithar, monk 202-203, 213-216, 218-220, 222-225, 227, 229

Wulfram, bishop of Sens 562
Wuling, king of Zhao state 595

Xi Wu, general of the Wei state 581

Xradešahr/Jesus (Šābuhragān) 186-187, 190

Yafeth (OT) 401

Yahweh 391-392, 768

Yaḥyā, son of Muḥammad Fațịh 433

Yahyā I-Maghāmi, student of Ibn Ḥabīb 349

Ya'jūj wa-Ma'jūj see Gog and Magog

Yama, Hindu deity 588, 592-596, 598-599, 617-622, 624-626

Yaśas, mythical king of Shambala 145

Yaśomitra, Indian Buddhist author 150

Yavana, foreign king-invador in Buddhist eschatology and Sanskrit name for the Greeks 362

Ye she mtsho rgyal, consort of Padma-

sambhava 359

Yoshida Kaneatsu, Shintō priest 541

Yoshida Kanetomo, Shintō theologian 541

Yuan, dynasty 370

Yuan, emperor 370

Zacharias, pope 58

Zaradusht/Zarathustra 186, 405

Zeus, Greek deity 272

Zillah, wife of Lamech (OT) 266

Zoan, Pharaoh 263 